\title{
Relationship between cognition and the severity of chronic tinnitus: Validation of the Japanese version of the Tinnitus Cognitions Questionnaire
}

\author{
Kayoko Kabaya ${ }^{1 *}$ \\ Mariko Takahashi1 ${ }^{1}$ \\ Yuji Sato ${ }^{2}$ \\ Kenichi Sekiya ${ }^{3}$ \\ Shinobu Ito ${ }^{4}$ \\ Shinichi Iwasaki
}

\begin{abstract}
Objective: The Tinnitus Cognitions Questionnaire (TCQ) is a scale designed to assess the positive and negative cognitions associated with tinnitus. The purpose of this study was to validate the Japanese version of the TCQ and to analyze the relationship between cognition and the severity of chronic tinnitus.

Methods: This was a cross-sectional, multicenter study. Patients with chronic tinnitus persisting for longer than 3 months were included. Participants completed the TCQ, the Tinnitus Handicap Inventory (THI), and the Hospital Anxiety and Depression Scale (HADS). They also completed the TCQ a second time 3-7 days later. The questionnaire was translated into Japanese. A factor analysis was performed and the convergent and discriminant validity, internal consistency, and test-retest reliability were evaluated.

Results: The total sample consisted of 75 participants. We obtained high Cronbach's $\alpha$ coefficients for the total score and subscales, ranging from 0.933 to 0.974 . The total score and subscale interclass correlation coefficients for test-retest reliability ranged from 0.631 to 0.963 . The factor analysis yielded a two-factor structure: negative and positive subscales. The convergent and discriminative validity was sufficiently clear. The negative subscale of the TCQ was strongly correlated with the THI and the HADS.

Conclusion: The Japanese version of the TCQ was validated here. It also exhibited a two-factor structure that was well matched with previous data. And it was a highly consistent and reliable measure that can be used to evaluate cognitions in patients with chronic tinnitus. Negative cognition for tinnitus was greatly related to handicap and psychological state.
\end{abstract}

Keywords: Tinnitus; Tinnitus Cognitions Questionnaire (TCQ); Tinnitus Handicap Inventory (THI); Hospital Anxiety and Depression Scale (HADS); Validity; Cognitions; Severity.

\footnotetext{
${ }^{1}$ Department of Otolaryngology-Head and Neck Surgery, Nagoya City University Graduate School of Medical Science, Aichi, Japan

2Department of Otolaryngology-Head and Neck Surgery, Kasugai Municipal Hospital, Aichi, Japan

${ }^{3}$ Department of Otolaryngology-Head and Neck Surgery, Anjo Kosei Hospital, Aichi, Japan

${ }^{4}$ Department of Otolaryngology-Head and Neck Surgery, Toyohashi Municipal Hospital, Aichi, Japan

Department of Otolaryngology-Head and Neck Surgery, Nagoya City University Graduate School of Medical Science, 1 Kawasumi, Mizuho-cho, Mizuho-ku, Nagoya, Aichi, 467-8601, Japan. E-mail: kabayakayoko@gmail.com. Phone: +81-52-853-8256

Paper submitted on May 03, 2021; and Accepted on June 28, 2021
} 


\section{INTRODUCTION}

Various questionnaires have been developed and validated for the assessment of tinnitus complaints. The Tinnitus Cognitions Questionnaire (TCQ) is a scale that was designed to assess the positive and negative cognitions associated with tinnitus ${ }^{1}$. In the treatment of chronic tinnitus, cognitive behavioral therapy is recommended by the American Academy of Otolaryngology- Head and Neck Surgery (AAO-HNS) guidelines ${ }^{2}$. Cognitive behavioral therapy is needed in patients who have many negative thoughts and few positive thoughts regarding tinnitus ${ }^{1}$ therefore, it is important to evaluate changes in cognition for tinnitus before, during, and after cognitive behavioral therapy. However, only English-language versions of the questionnaires that evaluate cognition for tinnitus exist.For the evaluation of the severity of chronic tinnitus, the Tinnitus Handicap Inventory $(\mathrm{THI})^{3}$, Tinnitus Questionnaire $(\mathrm{TQ})^{4}$, Tinnitus Handicap Questionnaire $(\mathrm{THQ})^{5}$, and Tinnitus Functional Index $(\mathrm{TFI})^{6}$ are available in various languages. For the evaluation of anxiety and depression, the Hospital Anxiety and Depression Scale $(\mathrm{HADS})^{7}$, Self-rating Depression Scale $(\mathrm{SDS})^{8}$, and State-Trait Anxiety Inventory (STAI) ${ }^{9}$ are also available in various languages. Conversely, the TCQ and Tinnitus Acceptance Questionnaire (TAQ) ${ }^{10}$ have been prepared for the evaluation of tinnitus thought and cognition, but, at present, only the English versions are available, and no studies of these issues have been performed in other languages and other cultures. The evaluation of the validity of questionnaires of cognitions for tinnitus in other languages and cultures is urgently required. The purpose of this study was to validate the Japanese version of the TCQ and to analyze the relationship between cognition and the severity of handicap as well as the psychological evaluation of chronic tinnitus.

\section{MATERIALS AND METHODS}

Study design: This was a cross-sectional study that was performed at three secondary care hospitals and one tertiary care hospital via psychometric evaluation. The ethical committee of the Nagoya City University Graduate School of Medical Sciences (identification number: 60-17-0065) and similar ethical review boards at all other study sites approved the study, which was carried out according to the guidelines set by the Helsinki Declaration. Written informed consent was obtained from all participants.

Subjects: Participants were recruited from the otolaryngology departments of the various participating hospitals from December 1, 2017 to September 30, 2018. Eligible participants included outpatients with chronic tinnitus persisting for longer than 3 months. All participants were at least 20 years old and native Japanese speakers. We excluded patients who could not answer the questionnaires because of old age, blindness, cognitive decline, neurodegenerative diseases, or psychiatric diseases.
Methods: The participants filled the following three questionnaires at the hospital: TCQ, THI, and HADS. For the re-test study, the participants filled the TCQ again at home 3-7 days after the initial session. The participants returned the re-test questionnaire by mail or in person. In this study, we used the Japanese version of the three questionnaires.

Tinnitus Cognitions Questionnaire (TCQ): The TCQ is a 26 -item, self-reported questionnaire that evaluates cognitions for chronic tinnitus. The first group of 13 items refers to negative thoughts and the second group of 13 items refers to positive thoughts. The participants were asked to indicate how often they were aware of thinking each thought while noticing tinnitus. The responses were marked on a 5-point scale offering the options of "never" $=0$, "rarely" $=1$, "occasionally" $=2$, "frequently" $=3$, or "very frequently" $=4$. Positive items were reverse scored; thus, a high score on the positive subscale indicates a low level of positive thinking. The minimum total score is 0 and the maximum total score is 104 . The reliability and validity of the TCQ have been documented in the original version ${ }^{1}$.

Translation-re-translation of the TCQ: The bilingual translators performed the forward translation. The translator had a medical background, and his/her mother tongue was Japanese. The other bilingual speaker without a medical background performed the back-translation. An expert team composed of one of the investigators and a bilingually experienced translator reviewed the preliminary version of the TCQ. Testing was performed on a sample of randomly chosen patients, for face validity. We subjected all patients to a structured interview after they answered the inventory. We asked them whether they had any comments regarding the content or layout of the inventory. According to the answers obtained from the interview and face validity test, we performed minor alterations, and our Japanese version of the TCQ was finalized.

Tinnitus Handicap Inventory (THI): The THI is a 25 -item, self-reported questionnaire that evaluates the impact of tinnitus on quality of life. Itcomprises a Functional subscale, an Emotional subscale, and a Catastrophic subscale, which contain ${ }^{11}$, nine, and five items, respectively, scored on a scale of 4 points for "Yes", 2 points for "Sometimes", and 0 point for "No". The total score ranges from 0 to 100 points, with a higher total score reflecting a more severe handicap. The reliability and validity of the THI have been documented for both the original and the Japanese versions of this questionnaire.

Hospital Anxiety and Depression Scale (HADS): The HADS is a 14-item scale that evaluates general anxiety and depression in patients in a non-psychiatric hospital setting. It comprises an anxiety subscale and a depression subscale, which both contain seven items scored on a 4-point scale. Higher subscale scores indicate higher levels of either anxiety or depression. The 
reliability and validity of the HADS have been documented for both the original and the Japanese versions of the questionnaire ${ }^{12,13}$. Validity-reliability analysis and statistical analysis: We conducted a factor analysis. We assessed the intraclass consistency coefficients (ICCs) for the two subscales: negative and positive thinking of tinnitus. The internal consistency regarding reliability of the TCQ was assessed using Cronbach's alpha. The test reliability was assessed. We calculated Pearson's correlation coefficients for the total item score and the score for two subscales and ICCs. The convergent and discriminant validities were evaluated using the $\mathrm{THI}$ and HADS results.EZR ver. 1.24 (Saitama Medical Center, Jichi Medical University, Saitama, Japan) ${ }^{14}$, which is a graphical user interface for R, and HAD16, which is a statistical free software, were used for evaluating the data.

\section{RESULTS}

A total of 95 patients answered the questionnaires. After excluding cases with missing values, the total sample of the study comprised 75 participants (79.0\%). We found no influential outliers and no systematic differences between the total sample and the excluded cases. We assessed 32 males (42.7\%) and 43 females (57.3\%) with an average age of 63.8 years (standard deviation, 12.3 years; range, 37-85 years). The breakdown of the sample according to the three facilities was as follows: 47 patients at the tertiary care hospital and 28 at the secondary care hospitals (18 in urban areas and 10 in suburbs). The average, standard deviation, and range of the scores on the TCQ, THI, and HADS are shown in Table 1.
Validity-reliability analysis: For the assessment of internal consistency reliability, Cronbach's alpha was 0.933 for the total score on the TCQ, 0.974 for the negative subscale, and 0.950 for the positive subscale. test reliability was assessed as a second investigation in 75 patients. The ICCs for the total score and two subscales are shown in Table 2. The ICC of the total score was rather high (0.963) and the ICCs of the negative and positive subscales were lower than that of the total score $(0.631$ and 0.679 , respectively).For the assessment of structural validity, the optimal number of factors was two. We assessed the factor pattern and factor structure matrix using Promax rotation (Table 3). One of two factors corresponded exactly to the negative subscale, whereas the other one corresponded to the positive subscale.

\section{Relationship between cognition and other psychometric properties in patients with tinnitus}

Pearson's correlation coefficient was used to assess the convergent/discriminant validity, as shown in Table 4. There was a weak correlation between the negative and positive subscales of the TCQ. The negative subscale score of the TCQ was strongly correlated with the total score and the scores on the three subscales of the THI $(0.438-0.656)$, whereas moderate correlations were detected between the scores on the depression and anxiety subscales of the HADS (0.416 and 0.450 , respectively). Conversely, the positive-subscale score of the TCQ was weakly correlated with the total score and the scores on the three subscales of $\mathrm{THI}$ and on the depression and anxiety subscales of the HADS (0.009-0.162).

Table 1: Average, standard deviation, and range of the scores on the TCQ, THI, and HADS.

\begin{tabular}{cccc}
\hline & Average & Standard deviation & Range \\
\hline First test & & & 14.8 \\
TCQ total & 46.6 & 12.4 & $0-78$ \\
TCQ negative & 20.3 & 11.3 & $0-52$ \\
TCQ positive & 26.3 & $0-52$ \\
Re-test & & & $1-80$ \\
TCQ total & 46.1 & 14.1 & $0-51$ \\
TCQ negative & 15.9 & 13.6 & $0-52$ \\
TCQ positive & 30.1 & 14.3 & $0-98$ \\
THI total & 34.8 & 22.2 & $0-42$ \\
THI functional & 16.3 & 9.8 & $0-36$ \\
THI emotional & 11.1 & 9.0 & $0-20$ \\
THI catastrophic & 7.4 & 4.9 & $0-30$ \\
HADS total & 11.5 & 7.6 & $0-16$ \\
HADS depression & 5.4 & 3.7 & $0-18$ \\
HADS anxiety & 6.1 & 4.5 & \\
\hline
\end{tabular}

TCQ, Tinnitus Cognitions Questionnaire

THI, Tinnitus Handicap Inventory

HADS, Hospital Anxiety and Depression Scale

Table 2: Intraclass correlation coefficients for test-retest reliability.

$\begin{array}{cc}\text { TCQ total } & 0.963[0.491-0.989] \\ \text { TCQ negative } & 0.631[-1.006-0.864] \\ \text { TCQ positive } & 0.679[-0.445-0.874]\end{array}$


Table 3: Factor matrix, commonality, and interfactor correlation in the factor analysis.

\begin{tabular}{|c|c|c|c|c|}
\hline & Outline of items & Factor 1 & Factor 2 & Commonality \\
\hline \multicolumn{5}{|c|}{ Negative subscale } \\
\hline 8 & I think "How can I go on putting up with this noise?" & .874 & .052 & .770 \\
\hline 12 & I think "The noise will overwhelm me" & .847 & -.072 & .717 \\
\hline 7 & I think "I can't enjoy what I'm doing because of the noise" & .838 & .069 & .712 \\
\hline 10 & I think "Why can't anyone help me?" & .837 & -.010 & .700 \\
\hline 4 & I think "The noise makes my life unbearable." & .830 & -.025 & .689 \\
\hline 2 & I think "Why me? Why do I have to suffer this horrible noise?" & .807 & -.002 & .650 \\
\hline 3 & I think "What did I do to deserve this?" & .765 & .043 & .589 \\
\hline 9 & I think "The noise will drive me crazy" & .750 & -.021 & .561 \\
\hline 13 & I think "With this noise, life is not worth living" & .749 & -.002 & .560 \\
\hline 6 & I think "If only I could get some peace and quiet" & .743 & .118 & .574 \\
\hline 5 & I think "Nobody understands how bad the noise is" & .703 & .028 & .497 \\
\hline 1 & I think "If only the noise would go away" & 647 & -.069 & .420 \\
\hline 11 & I think "My tinnitus is never going to get better" & .593 & -.033 & .351 \\
\hline \multicolumn{5}{|c|}{ Positive subscale } \\
\hline 19 & I think "The noise will eventually get less annoying if I try to distract myself" & -.080 & .816 & .666 \\
\hline 23 & I think "The noise might be there, but I can still enjoy things" & -.317 & .737 & .624 \\
\hline 22 & I tell myself "I can learn to live with it" & .149 & .709 & .534 \\
\hline 16 & I think "I'll be able to enjoy things if I keep my attention off the noise" & .056 & .705 & .504 \\
\hline 18 & I think "There are things in life worse than tinnitus" & .074 & .670 & .459 \\
\hline 25 & I tell myself "I won't think about the noise" & .162 & .626 & .426 \\
\hline 26 & I think "The noise is a nuisance but I just won't let it bother me" & -.054 & .621 & .385 \\
\hline 21 & I say to myself "It will help if I try to think of something pleasant" & .146 & .596 & .384 \\
\hline 17 & I think "I'm not the only person with tinnitus" & -.083 & .561 & .318 \\
\hline 24 & I tell myself "Think of something else other than the noise" & .217 & .541 & .349 \\
\hline 14 & I think "No matter how unpleasant the noise gets, I can cope" & -.028 & .516 & .265 \\
\hline 15 & I think "The noise might be unpleasant, but it won't drive me crazy" & -.014 & .509 & .259 \\
\hline 20 & I think "I have coped with the noise before, so I can cope again this time" & -.289 & .482 & .304 \\
\hline & Interfactor correlation & 0.42 & & \\
\hline
\end{tabular}

Table 4: Construct validity: Pearson's correlation coefficients between variables.

\begin{tabular}{|c|c|c|c|c|c|c|c|c|c|c|c|c|c|c|c|c|}
\hline & TCQ-T & & TCQ-N & & TCQ-P & & THI-T & & THI-F & & THI-E & & THI-C & & HADS-D & HADS-A \\
\hline \multicolumn{17}{|l|}{ TCQ-T } \\
\hline TCQ-N & .711 & ** & & & & & & & & & & & & & & \\
\hline TCQ-P & .641 & ** & -.083 & & & & & & & & & & & & & \\
\hline THI-T & .526 & ** & .656 & ** & .030 & & & & & & & & & & & \\
\hline THI-F & .445 & ** & .586 & ** & -.009 & & .939 & ** & & & & & & & & \\
\hline THI-E & .508 & ** & .643 & ** & .018 & & .956 & $\star \star$ & .832 & ** & & & & & & \\
\hline THI-C & .472 & ** & .438 & ** & .191 & * & .659 & ** & .539 & ** & .610 & $* \star$ & & & & \\
\hline HADS-D & .435 & ** & .416 & ** & .162 & * & .544 & $* *$ & .494 & ** & .540 & ** & .444 & $\star *$ & & \\
\hline HADS-A & .358 & ** & .450 & ** & .016 & & .537 & $\star \star *$ & .475 & $* *$ & .551 & $* \star$ & .329 & $\star \star$ & .681 & $\star \star *$ \\
\hline
\end{tabular}

** $\mathrm{P}<0.001$, * $\mathrm{P}<0.05$

TCQ-T/N/P, TCQ total/ negative subscale/positive subscale;

$\mathrm{THI}-\mathrm{T} / \mathrm{F} / \mathrm{E} / \mathrm{C}, \mathrm{THI}$ total/functional subscale/emotional subscale/catastrophic subscale;

HADS-D/A, HADS-depression subscale/anxiety subscale

\section{DISCUSSION}

The TCQ contributes to knowledge about the type and frequency of thoughts in relation to tinnitus. The English version of the TCQ was previously validated in 200 individuals from Australia and 342 individuals from the United Kingdom ${ }^{15,16}$. The findings of the present study provided support for the use of the TCQ in tinnitus research and in clinical practice in Japan, in a setting with different languages and cultures. The Japanese version of the TCQ also exhibited a two-factor structure, and negative cognition for tinnitus was greatly related to handicap and psychological state, whereas positive cognition for tinnitus was an independent thought process.Tinnitus is an auditory sensation that can cause discomfort or even pain; moreover, patients with tinnitus generally 
live with their problem for long periods ${ }^{17}$. Some patients with severe chronic tinnitus need cognitive behavioral therapy. When performing cognitive behavioral therapy, it is necessary to evaluate the patient's cognition for tinnitus and the changes in cognition after treatment. The TCQ is useful to assess the patient's cognition regarding tinnitus. The Japanese version of the TCQ proved to be a credible tool for the evaluation of tinnitus cognitions. The internal consistency ratio was sufficiently high. In the test-retest, the total score was sufficiently high; however, the scores on the positive and negative subscales were lower than the total scores of the TCQ. The Japanese version of the TCQ also exhibited a two-factor structure, and there was no correlation between the negative and positive subscales; therefore, changes in the negative and positive subscales were considered to occur independently. Handscomb et al. reported that negative thinking appeared to be associated with more problematic tinnitus ${ }^{16}$. This study also showed that patients with a severe handicap for tinnitus had more negative cognition. Considering these facts, to reduce the handicap for tinnitus, it is important to educate and counsel patients to decrease their negative thoughts for tinnitus. A previous study stated that positive thinking was not associated with unproblematic tinnitus, and the highest mean score on the positive subscale (indicating fewer frequent positive thoughts) was obtained for patients with no problem for tinnitus. This study also showed that positive cognition was weakly correlated with handicap for tinnitus, whereas there was no relationship between low frequency of positive thoughts and no handicap for tinnitus. Over time, and after improvement to the no-handicap condition, patients may have less frequent thought of both negative and positive nature. Therefore, the changes in cognition for tinnitus in the TCQ after treatment should be clarified in the future. Regarding the relationship between cognition for tinnitus and psychological state, Wilson et al. reported that depression evaluated by the Beck Depression Inventory was strongly correlated with the negative subscale and weakly correlated with the positive subscale. In this study, similar results were obtained for depression. Patients with many negative thoughts needed to be aware of their depression. Conversely, the relationship between TCQ scores and anxiety has not been reported. In this study, anxiety also exhibited a strong correlation with the negative subscale and a weak correlation with the positive subscale. The positive subscale of the TCQ was suggested to be an evaluation point that is independent of tinnitus handicap and psychological state. The discriminatory validity and convergent validity with respect to the TCQ and the THI and HADS have been demonstrated. This study had several limitations. The first limitation concerns the interval between the first test and the retest; the risk of recall bias cannot be ruled out because of this short interval. In the setting of this study, retest was set to 3-7 days after the first session, because the state of tinnitus in these patients changed easily and was considered to be the limitation of this test. Validation of the TCQ examination in a non-hospital environment or in the general population is an important next step of this research. Second, the present study was conducted among a population in a secondary and tertiary care context in Japan using the Japanese version of the TCQ. Therefore, further research is required in other languages and other populations. Finally, we did not evaluate the changes in the TCQ over time, or the responsiveness or interpretability of the TCQ in this study. Additional studies are required to examine these aspects.

\section{CONCLUSION}

The first validation of the TCQ in a language other than English for the evaluation of tinnitus cognitions was reported here. The Japanese version of the TCQ also exhibited a two-factor structure that was well matched with previous data; moreover, the Japanese version of the TCQ was a highly consistent and reliable measure that can be used to evaluate cognitions in patients with chronic tinnitus. Negative cognition for tinnitus was strongly correlated with handicap and psychological state, whereas positive cognition for tinnitus was an independent thought process.

\section{CONFLICT OF INTEREST}

The authors declares no conflict of interest.

\section{ACKNOWLEDGEMENT}

The authors would like to thank Enago (www.enago.jp) for the English language review.

\section{STATEMENT OF ETHICS}

This study was approved by the Research Ethics Committee, Graduate School of Medicine, at Nagoya City University (60-17-0065) and was conducted according to the tenets of the Declaration of Helsinki. A written informed consent was made by signing the questionnaire.

\section{FUNDING SOURCES}

This study was supported by JPSP KAKENHI Grant Number JP17K11338, 20K11161.

\section{REFERENCES}

1. Wilson PH, Henry JL. Tinnitus Cognitions Questionnaire: Development and Psychometric Properties of a Measure of Dysfunctional Cognitions Associated with Tinnitus. Int Tinnitus J. 1998;4:23-30.

2. Tunkel DE, Bauer CA, Sun GH, Rosenfeld RM, Chandrasekhar SS, Cunningham Jr ER, et al. Clinical practice guideline: tinnitus. Otolaryngology-Head and Neck Surgery. 2014;151:S1-S40.

3. Newman CW, Jacobson GP, Spitzer JB. Development of the Tinnitus Handicap Inventory. Arch Otolaryngol Head Neck Surg. 1996;122:143-8.

4. Meeus O, Blaivie C, Van de Heyning P. Validation of the Dutch and the French version of the Tinnitus Questionnaire. B-ENT. 2007;3 Suppl 7:11-7. 
5. Kuk FK, Tyler RS, Russell D, Jordan H. The psychometric properties of a tinnitus handicap questionnaire. Ear Hear. 1990;11:434-45.

6. Meikle MB, Henry JA, Griest SE, Stewart BJ, Abrams HB, McArdle R, et al. The tinnitus functional index: development of a new clinical measure for chronic, intrusive tinnitus. Ear Hear. 2012;33:153-76.

7. Zigmond AS, Snaith RP. The hospital anxiety and depression scale. Acta Psychiatr Scand. 1983;67:361-70.

8. Zung WW. A Self-Rating Deppression Scale. Arch Gen Psychiatry. 1965;12:63-70.

9. Spielberger CD, Gorsuch RL, Lushene RE, Vagg PR, Jacobs GA. Manual for the State-Trait Anxiety Inventory STAI (Form Y). Palo Alto, CA: Consulting Psychologists Press.1983.

10. Weise C, Kleinstäuber M, Hesser H, Westin VZ, Andersson G. Acceptance of tinnitus: validation of the tinnitus acceptance questionnaire. Cognitive behaviour therapy. 2013;42:100-15.

11. Omasa $H$, Kanzaki S, Takahashi M, Sato $H$, Wada T, Wada $T$, et al. Reliability and validation of the Tinnitus Handicap Inventory. Audiology Japan. 2019;62:607-14.
12. Matsudaira $\mathrm{T}$, Igarashi $\mathrm{H}$, Kikuchi $\mathrm{H}$, Kano $\mathrm{R}$, Mitoma $\mathrm{H}$, Ohuchi K, et al. Factor structure of the Hospital Anxiety and Depression Scale in Japanese psychiatric outpatient and student populations. Health Qual Life Outcomes. 2009;7:42.

13. Bjelland I, Dahl AA, Haug TT, Neckelmann D. The validity of the Hospital Anxiety and Depression Scale. An updated literature review. J Psychosom Res. 2002;52:69-77.

14. Kanda $Y$. Investigation of the freely available easy-to-use software 'EZR' for medical statistics. Bone Marrow Transplant. 2013;48:452-8.

15. Shimizu $H$. An introduction to the statistical free software HAD: Suggestions to improve teaching, learning and practice data analysis.: Journal of Media, Information and Communication; 2016. p. 59-73.

16. Handscomb LE, Hall DA, Shorter GW, Hoare DJ. Positive and Negative Thinking in Tinnitus: Factor Structure of the Tinnitus Cognitions Questionnaire. Ear Hear. 2017;38:12632.

17. Takahashi M, Kabaya K, Sekiya K, Matsuda F, Sekiya $Y$, Esaki S, et al. An improved system for grading and treating tinnitus. Auris Nasus Larynx. 2018;45:711-7. 